\title{
RUDIN-SHAPIRO SEQUENCES FOR ARBITRARY COMPACT GROUPS
}

\author{
For George. Szekeres on his sixty-fifth birthday \\ J. R. MCMULLEN and J. F. PRICE
}

(Received 26 August 1975)

\begin{abstract}
Let $G$ be a compact group. A sequence $\left\{f_{n}\right\}_{n=1}^{\infty}$ of functions in $L^{\infty}(G)$ is said to be a Rudin-Shapiro sequence (briefly, an RS-sequence) if the following conditions hold:

$$
\begin{aligned}
& \inf _{n}\left\|f_{n}\right\|_{2}>0 ; \\
& \sup _{n}\left\|f_{n}\right\|_{\infty}<\infty ; \\
& \lim _{n}\left\|\hat{f}_{n}\right\|_{\infty}=0 ;
\end{aligned}
$$

The main purpose here is to prove the following theorem:

THEOREM. Let $G$ be an infinite compact group. Then $G$ has an $R S$-sequence consisting of trigonometric polynomials.

The proof is carried out in section 1 while in section 2 several applications are given concerning set-theoretic relations between certain function spaces in harmonic analysis. The existence of RS-sequences for infinite LCA groups is well-known.
\end{abstract}

Notation. Let $G$ be a compact group. The Banach space of all continuous complex-valued functions on $G$ we denote by $C(G)$, and the Banach space of all complex Radon measures on $G$ by $M(G) ; L^{1}(G)$ will be identified in the usual way with the ideal in $M(G)$ of measures that are absolutely continuous with respect to normalized Haar measure $\lambda_{G}$.

The symbol $\hat{G}$ will denote a maximal set of pairwise inequivalent continuous irreducible unitary representations of $G$. The representation space of $\gamma \in G$ will be denoted by $H_{\gamma}$, and its dimension by $d_{\gamma}$. By $\mathbb{G}(\hat{G})$ we mean the linear space of all "sections" over $\hat{G}$, i.e. of all those functions 
$\Phi: \hat{G} \rightarrow \amalg_{\gamma \in \hat{G}} \mathscr{L}\left(H_{\gamma}\right)$ such that $\Phi(\gamma) \in \mathscr{L}\left(H_{\gamma}\right)$ for all $\gamma \in \hat{G}$. Here of course $\mathscr{L}\left(H_{\gamma}\right)$ is the von Neumann algebra of all (bounded) linear operators on $H_{\gamma}$. The Banach spaces $\mathfrak{S}^{p}(\hat{G})(1 \leqq p \leqq \infty)$ and $\mathcal{E}_{0}$, are defined as in (28.34) of Hewitt \& Ross (1970). The norms on the (F) $(\hat{G})$ are given by

$$
\begin{array}{ll}
\|\Phi\|_{\infty}=\sup \left\{\|\Phi(\gamma)\|_{\phi_{x}}: \gamma \in \hat{G}\right\} & \left(\Phi \in \mathscr{F}^{\infty}(\hat{G})\right) \\
\|\Phi\|_{p}=\left(\sum_{\gamma \in G} d_{\gamma}\|\Phi(\gamma)\|_{\phi_{p}}^{p}\right)^{1 / p} & \left(\Phi \in \mathscr{F}^{p}(\hat{G})\right)
\end{array}
$$

where \|\|$_{\phi_{p}}$ denotes the $p$ th von Neumann-Schatten norm on $\mathscr{L}\left(H_{\gamma}\right)$. In particular $\|A\|_{\alpha_{2}}=\left[\operatorname{tr}\left(A A^{*}\right)\right]^{\frac{1}{2}}$, and $\|A\|_{\phi_{\infty}}$ is the operator norm of $A$.

The Fourier-Stieltjes transform of $\mu \in M(G)$ we define as an element of (5) $(\hat{G})$ by

$$
\hat{\mu}(\gamma)=\int_{G} \gamma\left(x^{-1}\right) d \mu(x)
$$

and its Fourier series is the series (suitably interpreted)

$$
\mu \sim \sum_{\gamma \in G} d_{\gamma} \operatorname{tr}(\hat{\mu}(\gamma) \gamma(\cdot)) .
$$

The closure of the $n$th derived subgroup of $G$ we denote by $G^{(n)}$.

\section{Proof of the theorem}

Before commencing the proof we remark that the existence of RSsequences for infinite LCA groups is well-known; see Gaudry (1970) and (37.19b) of Hewitt \& Ross (1970). Also a weaker version is known to exist for infinite compact groups. Specifically, whenever $t \in] 2, \infty]$, a sequence $\left\{f_{n}\right\}_{n=1}^{\infty}$ of functions is said to be a $t$-RS-sequence if it satisfies conditions (1) and (3) of the definition of an RS-sequence with (2) replaced by

$$
\sup _{n}\left\|f_{n}\right\|_{r}<\infty .
$$

In Figà-Talamanca \& Price (1972), random Fourier series are used to show that t-RS-sequences with $t<\infty$ exist for all infinite compact groups. Also the existence of such sequences with other useful properties is demonstrated in Figà-Talamanca \& Price $(1972,1973)$. We have not been able to generalise these extra properties to RS-sequences.

Since the definition of an RS-sequence involves only three norms, it is easily verified that any RS-sequence may be replaced by an RS-sequence consisting of trigonometric polynomials. In this section we therefore prove merely the existence of an RS-sequence for any infinite compact group. 
Whenever the supports of the members of an RS-sequence are contained in some open set $U$, then we say that this sequence is a U-RS-sequence.

Our proof begins with two special cases, from which we proceed to deduce the general case.

(1.1) Proposition. (Gaudry (1970), Lemma 2.1). Let $G$ be an infinite compact abelian group and $U$ a nonvoid open subset of $G$. Then $G$ has a $U$ $R S$-sequence.

Now, let us say that a compact group $G$ is tall if for every positive integer $d$ there are at most finitely many elements of $\hat{G}$ of degree $d$.

(1.2) Proposition. Let $G$ be an infinite tall compact group and $U$ a nonvoid open subset of $G$. Then $G$ has a $U$-RS-sequence.

PROOF. The following construction depends on repeated applications of the fact that every measurable subset of $G$ of positive measure has a subset of half its measure.

Let $V \subseteq \bar{V} \subseteq U \subseteq G$ be measurable, $\lambda_{G}(V)=v>0$. Let $P_{1}, P_{2}$ be disjoint measurable subsets of $V$ such that $P_{1} \cup P_{2}=V, \lambda_{G}\left(P_{1}\right)=\lambda_{G}\left(P_{2}\right)$. Let $\pi_{1}=\left\{P_{1}, P_{2}\right\}$. If $\pi_{n-1}$ has been defined as a partition of $U$ into $2^{n-1}$ subsets $P_{n-1, i}\left(1 \leqq i \leqq 2^{n-1}\right)$ then form $\pi_{n}$ by writing $P_{n-1, i}$ as a disjoint union of two measurable subsets $P_{n, 2 i-1}, P_{n, 2 i}$ of equal measure. Thus $\pi_{n}$ is a set $\left\{P_{n, i}: 1 \leqq i \leqq\right.$ $\left.2^{n}\right\}$ of pairwise disjoint measurable subsets of $V$ of equal measure such that $V=\bigcup_{i=1}^{2 n} P_{n, i}$.

We now define a sequence of Rademacher functions associated with the sequence $\pi_{n}$. Put $r_{1}=\chi_{P_{i, 1}}-\chi_{P_{1,2},}$ and more generally let $r_{n}=\Sigma_{i=1}^{2 n}(-1)^{i+1} \chi_{P_{n, i}}$. Then $r_{n}$ takes values \pm 1 and

$$
\int_{P_{n-1, i}} r_{n} d \lambda_{G}=\int_{P_{n, 2 i-1}} r_{n} d \lambda_{G}+\int_{P_{n, 2 i}} r_{n} d \lambda_{G}=0
$$

(This construction is also used in Figà-Talamanca and Gaudry (1970).) Clearly we have $\left\|r_{n}\right\|_{p}=v^{1 / p}(1 \leqq p<\infty),\left\|r_{n}\right\|_{\infty}=1$, and $\int_{G} r_{m} r_{n} d \lambda_{G}=v \delta_{m n}$ for $m, n \in\{1,2, \cdots\}$. Define $f_{n}=v^{-\frac{1}{2}} r_{n}$; then we have

$$
\begin{aligned}
& \left\|f_{n}\right\|_{p}=v^{1 / p-\frac{1}{2}}(1 \leqq p<\infty), \\
& \left\|f_{n}\right\|_{\infty}=v^{-\frac{1}{2}}, \quad \text { and } \\
& \int_{G} f_{m} f_{n} d \lambda_{G}=\delta_{m n} \quad(m, n \in\{1,2, \cdots\}) .
\end{aligned}
$$

We claim that $\left\{f_{n}\right\}_{n=1}^{\infty}$ is an RS-sequence under the assumption that $G$ is tall. 
Indeed, in view of (4) all that is required is to show that $\left\|\hat{f}_{n}\right\|_{\infty} \rightarrow 0$ as $n \rightarrow \infty$. Now Parseval's formula for $G$ is

$$
\|f\|_{2}^{2}=\sum_{\gamma \in G} d_{\gamma} \operatorname{tr}\left(\hat{f}(\gamma) \hat{f}(\gamma)^{*}\right) \quad\left(f \in L^{2}(G)\right)
$$

Also, by Hewitt and Ross (1963 and 1970), (D.51) we have, for $A \in \mathscr{L}(H)$, $\operatorname{tr}\left(A A^{*}\right) \geqq\|A\|_{\phi_{\infty}}^{2}$, and hence

$$
\sum_{\gamma \in G} d_{\gamma}\left\|\hat{f}_{n}(\gamma)\right\|_{\phi_{\infty}}^{2} \leqq\left\|f_{n}\right\|_{2}^{2}=1
$$

This makes it clear for each $n \geqq 1$ and each $\varepsilon>0$, the set $\left\{\gamma \in \hat{G}:\left\|\hat{f}_{n}(\gamma)\right\|_{\phi_{\infty}}>\right.$ $\varepsilon\}$ is finite (this merely reproves the well known fact that $\mathfrak{F}^{2} \subseteq \mathfrak{E}_{0}$ ). Hence we may conclude that

$$
\left\|\hat{f}_{n}\right\|_{\infty}=\left\|\hat{f}_{n}\left(\gamma_{n}\right)\right\|_{\infty_{\infty}}
$$

for some $\gamma_{n} \in \hat{G}$. Let $\Delta=\left\{\gamma_{n}: n \geqq 1\right\}$. If $\Delta$ is infinite, then $d_{\gamma_{n}} \rightarrow \infty$ as $n \rightarrow \infty$, by our assumption about the representations of $G$. Hence by (5), we have

$$
\left\|\hat{f}_{n}\right\|_{\infty}=\left\|\hat{f}_{n}\left(\gamma_{n}\right)\right\|_{\phi_{\infty}} \leqq d_{\gamma_{n}}^{-1 / 2},
$$

showing that $\left\{f_{n}\right\}_{n=1}^{\infty}$ is an RS-sequence as asserted.

In any case, since $\left\{f_{n}\right\}_{n=1}^{\infty}$ is orthonormal in $L^{2}(G)$, it follows that $\left\|\hat{f}_{n}(\gamma)\right\|_{\phi_{\infty}} \rightarrow 0$ for each $\gamma \in \hat{G}$. Thus when $\Delta$ is finite, we have

$$
\left\|\hat{f}_{n}\right\|_{\infty} \leqq \sup _{\gamma \in \Delta}\left\|\hat{f}_{n}(\gamma)\right\|_{\phi_{\infty}} \rightarrow 0 \quad \text { as } \quad n \rightarrow \infty
$$

and again $\left\{\hat{f}_{n}\right\}_{n=1}^{\infty}$ is an RS-sequence. This completes the proof.

(1.3) Lemma. Let $\Gamma$ be a closed subgroup of the compact group $G$. Then there is a quasi-invariant normalised measure $\lambda$ on the coset space $G / \Gamma$ with the following property: if $f$ is a nonnegative extended real-valued $\lambda_{G}$-integrable function on $G$, then the set of cosets $x \Gamma$ in $G / \Gamma$ for which the function $\xi \rightarrow f(x \xi)$ $(\xi \in \Gamma)$ is not $\lambda_{\Gamma}$-integrable is $\lambda$-null; the function on $G / \Gamma$ defined $\lambda$-a.e. by $x \Gamma \rightarrow \int_{\Gamma} f(x \xi) d \lambda_{\Gamma}(\xi)$ is $\lambda$-integrable, and we have

$$
\int_{G} f(x) d \lambda_{G}(x)=\int_{G / \Gamma} \int_{\Gamma} f(x \xi) d \lambda_{\Gamma}(\xi) d \lambda(x \Gamma) .
$$

The reader is referred to Bourbaki (1963), Chapter VII, section 2, discussion following Théorème 2 .

(1.4) Lemma. Let $\phi: G \rightarrow G_{1}$ be a continuous surjective homomorphism, where $G$ and $G_{1}$ are compact groups and $G_{1}$ has an $R S$-sequence. Then $G$ has an $\mathrm{RS}$-sequence. 
This is proved in Edwards \& Price (1970), A.3.3.

(1.5) LeMMA. Let $\Gamma$ be a closed subgroup of a separable compact group $G$, and suppose that $\Gamma$ admits an RS-sequence. Then $G$ does also.

Proof. Since $G$ is separable, there is a Borel section $B$ for $\Gamma$ in $G$ (Mackey (1951)), i.e., a Borel set $B$ in $G$ which meets each coset $x \Gamma$ in exactly one point (we may assume $B \cap \Gamma=\{e\}$ ). Define a map $b: G / \Gamma \rightarrow B$ by setting $b(x \Gamma)$ as the unique member of $B \cap x \Gamma$. Let $h \in C(\Gamma)$. Put

$$
h^{0}(x \xi)=h(\xi) \quad(x \in B, \xi \in \Gamma) .
$$

The properties of $B$ ensure that $h^{0}$ is well-defined as an element of $L^{\infty}(G)$. Application of Lemma (1.3) shows immediately that

$$
\left\|h^{0}\right\|_{2}^{2}=\int_{G / \Gamma}\|h\|_{2}^{2} d \lambda(x)=\|h\|_{2}^{2}
$$

It is also easy to see that

$$
\left\|h^{0}\right\|_{\infty}=\|h\|_{\infty}
$$

Now, let $\sigma \in \hat{G}$ be fixed. Then the restriction $\left.\sigma\right|_{\Gamma}$ of $\sigma$ to $\Gamma$ admits a decomposition

$$
\left.\sigma\right|_{\Gamma}=\bigoplus_{\tau \in \Gamma} n_{\sigma}(\tau) \cdot \tau
$$

( $n_{\sigma}(\tau)$ is the multiplicity of $\tau$ in $\left.\sigma\right|_{\Gamma}$ and $n_{\sigma}(\tau)=0$ for all save finitely many $\tau$ ). This decomposition is given via some unitary intertwining transformation from $\bigoplus_{\tau \in \mathrm{r}} n_{\sigma}(\tau) H_{\tau}$ to $H_{\sigma}$ which by transport of structure gives rise to a (von Neumann) algebra isomorphism

$$
\alpha_{\sigma}: \bigoplus_{\tau \in \hat{\Theta}} n_{\sigma}(\tau) \mathscr{L}\left(H_{\tau}\right) \rightarrow \mathscr{L}\left(H_{\sigma}\right)
$$

Then we have by (1.3)

$$
\begin{aligned}
\left(h^{0}\right)^{\wedge}(\sigma) & =\int_{G} h^{0}(x) \sigma\left(x^{-1}\right) d \mu(x)=\int_{G / \Gamma}\left(\int_{\Gamma} h^{0}(x \xi) \sigma\left((x \xi)^{-1}\right) d \lambda_{\Gamma}(\xi)\right) d \lambda(x \Gamma) \\
& =\int_{G / \Gamma}\left(\int_{\Gamma} h(\xi) \sigma\left(\xi^{-1}\right) d \lambda_{\Gamma}(\xi)\right) \sigma\left[(b(x \Gamma))^{-1}\right] d \lambda(x \Gamma) \\
& =\int_{G / \Gamma}\left(\int_{\Gamma} h(\xi) \alpha_{\sigma}\left[\bigoplus_{\tau} n_{\sigma}(\tau) \cdot \tau\left(\xi^{-1}\right)\right] d \lambda_{\Gamma}(\xi)\right) \sigma\left[(b(x \Gamma))^{-1}\right] d \lambda(x \Gamma) \\
& =\alpha_{\sigma}\left(\bigoplus_{\tau} n_{\sigma}(\tau) \hat{h}(\tau)\right) \int_{G / \Gamma} \sigma\left(b(x \Gamma)^{-1}\right) d \lambda(x \Gamma) .
\end{aligned}
$$


It follows that we have

$$
\begin{array}{r}
\left\|\left(h^{0}\right)^{\wedge}(\sigma)\right\|_{\phi_{x}} \leqq\left\|\alpha_{\sigma}\left(\underset{\tau}{\bigoplus} n_{\sigma}(\tau) \hat{h}(\tau)\right)\right\|_{\phi_{x}}=\left\|\bigoplus_{\tau} n_{\sigma}(\tau) \hat{h}(\tau)\right\|_{\phi_{\infty}} \\
\left\|\left(h^{0}\right)^{\wedge}(\sigma)\right\|_{\phi_{x}} \leqq\left\|\alpha_{\sigma}\left(\underset{\tau}{\oplus} n_{\sigma}(\tau) \hat{h}(\tau)\right)\right\|_{\phi_{\infty}} \\
=\max \left\{\|\hat{h}(\tau)\|_{\phi_{x}}: n_{\sigma}(\tau) \neq 0\right\} \leqq\|\hat{h}\|_{\infty}
\end{array}
$$

and hence that $\left\|\left(h^{0}\right)^{\wedge}\right\|_{\infty} \leqq\|\hat{h}\|_{\infty}$. This combined with equalities (7) and (8) shows that if $\left\{h_{n}\right\}$ is an RS-sequence on $\Gamma$ (with the $h_{n}$ restricted to be continuous-see the opening remarks of this section) then $\left\{h_{n}^{o}\right\}$ is an RS-sequence on $G$.

(1.6) LEMMA. [M. F. Hutchinson, private communication]. Let $G$ be $a$ prosolvable group (i.e. a projective limit of finite solvable groups) in which each derived factor $G / G^{(n)}$ is finite. Then $G$ is tall.

Proof. Since $G$ is profinite it is totally disconnected. Let $\gamma \in \hat{G}$. Then $\gamma(G)$ must be finite since it is a totally disconnected compact Lie group. Furthermore, since $\gamma(G)$ is also prosolvable, it must be solvable. Let $d$ be the degree of $\gamma$.

By Zassenhaus (1938) there is a number $l>0$ depending on $d$ only such that the solvable length of $\gamma(G)$ is at most $l$. Hence, using the fact that $G / \mathrm{ker}$ $\gamma$ and $\gamma(G)$ are isomorphic, $G^{(l)} \leqq \operatorname{ker} \gamma$.

Now let $d$ be a fixed positive integer. The members of $\left\{\gamma \in \hat{G}: d_{\gamma}=d\right\}$ must all satisfy $G^{(l)} \subseteq \operatorname{ker} \gamma$ where $l=l(d)$ and hence this set corresponds under an obvious injective map to a subset of $\left(G / G^{(l)}\right)^{\wedge}$. But the latter set is finite by the hypothesis, and the lemma is proved.

(1.7) Conclusion of the Proof. Let $G$ be an infinite compact group. Then $G$ has an infinite separable compact quotient group [Hewitt \& Ross (1963), Theorem (8.7)]. Lemma (1.4) indicates that it is enough then to prove the theorem under the assumption that $G$ is separable.

According to McMullen (1974), $G$ either has an infinite abelian subgroup or an infinite closed topologically-2-generator pro- $p$ torsion subgroup ( $p$ an odd prime). In either case, let us call the subgroup in question $\Gamma$. In the first case, $\Gamma$ has an RS-sequence by proposition (1.1). In the second, the same conclusion follows from lemma (1.6) and proposition (1.2).

Since $G$ is separable, the theorem now follows from Lemma (1.5). 


\section{Applications}

Techniques for applying RS-sequences to problems in harmonic analysis are well-known. For example, see Hewitt \& Ross (1970), (37.19), Gaudry (1970), Edwards \& Price (1970) and Figà-Talamanca \& Price (1972).

Here we sketch the details of three applications.

Application A. (2.1) In the case of compact groups, the HausdorffYoung theorem states that $\hat{f} \in \overleftarrow{C}^{p}$ whenever $f \in L^{p}, 1 \leqq p \leqq 2$, and $1 / p+1 / p^{\prime}=1$. Thus if $f \in C(G)$, then $\hat{f} \in \mathbb{F}^{q}$ for all $q \in[2, \infty]$. When $G$ is infinite and abelian this is known to be best possible in the sense that there exists $f \in C(G)$ such that $\hat{f}$ belongs to no (s) for $q \in[1,2[$ (see Hewitt \& Ross (1973), (37.19(c)) where an extension of this result is given for all locally compact abelian groups).

(2.2) Proposition: Whenever $G$ is an infinite compact group there exists $f \in C(G)$ such that $\hat{f}$ belongs to no (5) for $q \in[1,2[$.

The proof will use the following lemma, the proof of which follows directly from the definitions of the ${ }^{p} p$ and their respective norms.

Lemma. If $\phi \in \mathfrak{F}^{p}$, where $1 \leqq p<\infty$, then $\phi \in \mathfrak{F}^{q}$ for all $p \leqq q \leqq \infty$ and moreover

$$
\|\phi\|_{q}^{q} \leqq\|\phi\|_{p}^{p}\|\phi\|_{\infty}^{q-p}
$$

holds for $p \leqq q<\infty$.

Proof of (2.2). Suppose that the statement of the proposition is not valid, that is, that $f \rightarrow \hat{f}$ defines a map from $C(G)$ into $\cup\left\{E^{a}: 1 \leqq q<2\right\}$. Let $\left\{q_{n}\right\}$ be a sequence in $[1,2[$ which approaches 2 monotonically; then $\cup\left\{\mathcal{C}^{q}: 1 \leqq q<2\right\}=\cup\left\{\mathfrak{E}^{a_{n}}: n \geqq 1\right\}$. A direct application of Edwards (1965), Theorem 6.5.5, with $E=\mathcal{F}^{2}, F=C(G), u=$ Fourier transform, $F_{n}=\left(\mathcal{F}_{n}\right.$ and $u_{n}$ the identity map on $F_{n}$ shows that there exists an integer $k$ such that $C(G)^{\wedge} \subseteq\left(\mathfrak{F}^{2} q_{k}\right.$. It now follows from the closed graph theorem that for some $K>0$ we have

$$
\|\hat{f}\|_{a_{k}} \leqq K\|f\|_{\infty}
$$

for all $f \in C(G)$. Let $f_{n}$ be an RS-sequence consisting of continuous functions. Then there exist $m, M>0$ such that

$$
m \leqq\left\|f_{n}\right\|_{2} \leqq\left\|f_{n}\right\|_{\infty} \leqq M
$$

for all $n \geqq 1$. From the preceding lemma, we have

$$
\left\|f_{n}\right\|_{2}^{2}=\left\|\hat{f}_{n}\right\|_{2}^{2} \leqq\left\|\hat{f}_{n}\right\|_{q_{k}}\left\|\hat{f}_{n}\right\|_{x}^{2-q_{k}}
$$


and so we have

$$
\begin{aligned}
\left\|\hat{f}_{n}\right\|_{q_{k}} & \geqq\left\|f_{n}\right\|_{2}^{2 / q_{k}} /\left\|\hat{f}_{n}\right\|_{\infty}^{\left(2-q_{k}\right) / q_{k}} \\
& \geqq m^{2 / q_{k}} /\left\|\hat{f}_{n}\right\|_{\infty}^{\left(2-q_{k}\right) / q_{k}} \rightarrow \infty \text { as } n \rightarrow \infty
\end{aligned}
$$

since $1 \leqq q_{k}<2$. But this contradicts (9), in view of the fact that $\left\|f_{n}\right\|_{\infty} \leqq M$, for $n \geqq 1$.

Application B. (2.3) The Fourier transform $f \rightarrow \hat{f}$ carries $M$ into $\left(^{\infty}, L^{1}\right.$ into $\mathfrak{F}_{0}$ and $L^{p}$ into $\mathscr{C}^{p^{\prime}}$ when $1<p<2$. It is known that these maps are surjective if and only if $G$ is finite [Hewitt \& Ross (1970), (37.4) and (37.19 (a))]; a direct proof of these facts follows from the existence of an RSsequence. The surjectivity of the maps is trivial when $G$ is finite.

(2.4) Proposition. Let $G$ be an infinite compact group. The images of $M(G), L^{1}(G)$ and $L^{p}(G)(1<p<2)$ under the Fourier transform are properly contained in $\mathfrak{F}^{\infty}, \mathfrak{F}_{0}$, and $\mathfrak{F}^{\circ}$ respectively.

Proof. The proofs are similar in detail to the second part of the proof of the proposition (2.2): one assumes the contrary, establishes an inequality analogous to (9), and obtains a contradiction by substituting therein the members of an RS-sequence.

Application C. (2.5) Given $p, q \in[1, \infty]$, then $\phi \in[5$ is said to be a $(p, q)$-multiplier if

$$
\sum_{\gamma \in \hat{G}} d_{\gamma} t r[\phi(\gamma) \hat{f}(\gamma) \gamma(\cdot)]
$$

is the Fourier series of a function in $L^{q}$ whenever $f \in L^{p}$ (an equivalent definition is available which makes sense for arbitrary locally compact groups). For example, it is well-known that $\hat{\mu}$ is a $(p, p)$-multiplier for all $p \in[1, \infty]$ whenever $\mu \in M(G)$. On the other hand, when $G$ is an infinite compact abelian group there exist functions in $F$ which are $(p, q)$-multipliers for all $p \in] 1, \infty]$ and all $q \in[1, \infty[$, which are not Fourier-Stieltjes transforms; see Brainerd \& Edwards (1966), Theorem (4.15). The proof is based upon the existence of an infinite Sidon set. In fact, given the existence of certain lacunary subsets of $\hat{G}$, examples of functions in $\mathfrak{F}_{5}$ with the preceding properties can easily be produced; c.f. (37.22) of Hewitt \& Ross (1970). However, there exist compact groups whose duals possess no reasonable lacunary sets. Suppose that a locally compact group has the property of possessing an RS-sequence of functions whose supports are contained in a fixed compact set. Then Theorem 5.7 of Edwards \& Price (1970) shows that for such groups there exists a $(p, q)$-multiplier for all pairs $(p, q)$ such that 
$1<p \leqq q<\infty$, but which is not a Fourier-Stieltjes transform. Since we have seen that every infinite compact group has an RS-sequence we have:

(2.6) Proposition. Let $G$ be an infinite compact group. There exists a function in (5) which is a $(p, q)$-multiplier for all pairs $(p, q)$ such that $1<p \leqq q<\infty$, but is not the Fourier-Stieltjes transform of any measure.

(2.7) Remarks (i). Proposition (2.6) improves Theorem 4.3 of FigàTalamanca \& Price (1972), the proof of which was based on the existence of $t$ RS-sequences, $t>x$, as defined above. As a consequence of the existence of these restricted RS-sequences, roughly all that could be shown was that there exist multipliers of the type under question which are not Fourier transforms of any element in $\cup\left\{L^{r}: 1<r \leqq \infty\right\}$.

(ii) Further cases of proposition (2.6) for infinite compact groups are accounted for by noting that all functions in $\mathcal{E}^{*}$ are $(p, q)$-multipliers when $1 \leqq q \leqq 2 \leqq p \leqq \infty$ (Table (36.20) of Hewitt \& Ross (1970)), whereas by proposition (2.4) there exist elements in $5^{\infty}$ which are not Fourier-Stieltjes transforms.

(iii) In the event of the existence of a $U$-RS-sequence where $U$ is some open subset of $G$ (see propositions (1.1) and (1.2)), application A can be strengthened to show that there exists $f \in C(G)$ with support in $\bar{U}$ such that $\hat{f}$ belong to no $\mathcal{F}^{a}$ with $q \in[1,2[$. Applications $\mathrm{B}$ and $\mathrm{C}$ can be improved in a like manner.

\section{References}

N. Bourbaki (1963), Éléments de Mathématique: Intégration, Chap. 7-8 (Hermann, Paris, 1963).

B. Brainerd and R. E. Edwards (1966), 'Linear operators which commute with translations, Part 1: representation theorems', J. Austral. Math. Soc. 6, 289-327.

R. E. Edwards (1965), Functional Analysis: Theory and Applications (Holt, Rinehart and Winston, New York, 1965).

R. E. Edwards and J. F. Price (1970), 'A naively constructive approach to boundedness principles, with applications to harmonic analysis', Enseignement Math. 16, 255-296.

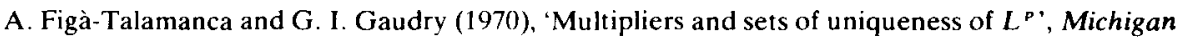
Math. J. 17, 179-191.

A. Figà-Talamanca and J. F. Price, (1972) 'Applications of random Fourier series over compact groups to Fourier multipliers', Pacific J. Math. 43, 531-541.

A. Figà-Talamanca and J. F. Price (1973), 'Rudin-Shapiro sequences on compact groups', Bull. Austral. Math. Soc. 8, 241-245.

G. I. Gaudry (1970), 'Bad behaviour and inclusion results for multipliers of type ( $p, q$ )', Pacific J. Math. 35, 83-94.

E. Hewitt and K. A. Ross (1963 and 1970), Abstract Harmonic Analysis (Vols I and II, Springer-Verlag, Berlin, 1963 and 1970).

G. W. Mackey (1951), 'On induced representations of groups', Amer. J. Math. 73, 576-592. 
J. R. McMullen (1974), 'Compact torsion groups' (Proceedings of the Second International Conference on the Theory of Groups, Lecture Notes in Mathematics No. 372, SpringerVerlag, Berlin, 1974).

H. Zassenhaus (1938), 'Beweis eines Satzes über diskrete Gruppen', Abh. Math. Sem. Univ. Hamburg 12, 289-312.

University of Sydney,

Sydney, N.S.W. 2006,

Australia

and

University of New South Wales,

Kensington, N.S.W. 2033,

Australia 